\begin{tabular}{|l|l|l|l|l|l|}
\hline $\begin{array}{l}\text { Nereis. Revista Iberoamericana } \\
\text { Interdisciplinar de Métodos, } \\
\text { Modelización y Simulación }\end{array}$ & 13 & $85-97$ & $\begin{array}{c}\text { Universidad Católica de } \\
\text { Valencia San Vicente Mártir }\end{array}$ & $\begin{array}{c}\text { Valencia } \\
\text { (España) }\end{array}$ & \begin{tabular}{l} 
ISSN 1888-8550 \\
\hline
\end{tabular}
\end{tabular}

\title{
DRP1 Mediated Fission Has an Important Role in Alzheimer's and Parkinson's Diseases
}

\author{
La fisión mediada por DRP1 tiene un papel importante \\ en las enfermedades de Alzheimer y Parkinson
}

Fecha de recepción y aceptación: 9 de febrero de 2021 y 22 de abril de 2021

DOI: $10.46583 /$ nereis_2021.13.802

Prieto J.A. ${ }^{\mathbf{1 , 2}}$, Gómez-Reino J. ${ }^{\mathbf{1}}$, Antón O. ${ }^{\mathbf{1}}$ and Ventura. I ${ }^{\mathbf{1 , 2 *}}$
${ }^{1}$ Faculty of Medicine and Health Science, Universidad Cátolica de Valencia San Vicente Mártir.
${ }^{2}$ Centro de Investigación Traslacional San Alberto Magno (CITSAM). Universidad Cátolica de Valencia San Vicente Mártir.
${ }^{*}$ Correspondencia: Universidad Católica de Valencia San Vicente Mártir. Faculty of Medicine and Health Science, Calle Quevedo, 2
46001 Valencia. Spain. E-mail: ignacio.ventura@mail.ucv.es

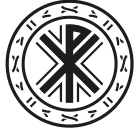

Universidad Católica de Valencia San Vicente Mártir

\section{ABSTRACT}

Objectives: Our objectives were to perform a bibliometric analysis with which to highlight the role of Drp1 in the pathogenesis of $\mathrm{AD}$ and $\mathrm{PD}$ as a fundamental regulator of mitochondrial fusion and fission homeostasis, in addition to highlighting Drp1 as a possible therapeutic target in AD and PD.

Methods: We carried out a bibliometric study based on a search in the PubMed database, selecting articles in the period from $01 / 01 / 2008$ to $12 / 31 / 2019$. The relationship of Drp 1 with AD and PD was explored through an analysis of the data and their representation in graphs.

Results: The analyzed data showed a slow but stable growth of interest in the relationship of Drp1 with AD and PD. First, we analyzed the number of publications per year and found that both the AD and PD remained stable over time. Next, we reviewed the quantitative validity of these data, expressed as the average JCR per year and the number of citations of articles showing an interest in the role of Drp1 in AD and PD.

Conclusions: Our bibliometric analysis showed that the relationship of Drp1 with AD and PD was significant, that interest was currently active and that Drp1 is regarded as a key protein in the pathogenesis of both diseases. Therefore, it is a possible therapeutic target for the treatment of both pathologies.

KEYWORDS: Drp1 (Dynamin related protein 1), AD (Alzheimer's disease), PD (Parkinson's disease), apoptosis, neurodegenerative disease.

\section{RESUMEN}

Objetivos: Nuestros objetivos son realizar un análisis bibliométrico con el que destacar la relevancia de Drp1 en la patogénesis de la EA y la EP como regulador fundamental de la homeostasis de la fusión y fisión mitocondrial, además de destacar a Drp1 como posible diana terapéutica en la EA y la EP.

Métodos: Hemos realizado un estudio bibliométrico a partir de una búsqueda en la base de datos PubMed, de los artículos seleccionados que se encuentran en el periodo comprendido entre el 
01/01/2008 y el 31/12/2019. Mediante un análisis de los datos y una representación en gráficos mostramos la relación de la Drp1 con la EA y la EP.

Resultados: Los datos analizados muestran un crecimiento lento pero estable del interés por la relación de la Drp1 con la EA y la EP. En primer lugar, analizamos el número de publicaciones por año que tanto la EA como la EP se mantienen estables a lo largo del tiempo, seguido del número de publicaciones que nos interesa observar la validez cuantitativa de estos datos, expresada en la media del JCR por año y el número de citas de los artículos, que muestran un interés por el papel de Drp1 en la EA y la EP.

Conclusiones: Nuestro análisis bibliométrico muestra que la relación de la Drp1 con la EA y la EP es relevante, que tiene interés en la actualidad y que están aplicando la Drp1 como proteína clave en la patogénesis de ambas enfermedades y, por tanto, como posible diana terapéutica para el tratamiento. de ambas patologías.

PALABRAS CLAVE: Drp1 (proteina relacionada con la dinamina 1), EA (enfermedad de Alzheimer), EP (enfermedad de Parkinson), apoptosis, enfermedad neurodegenerativa.

\section{INTRODUCTION}

Mitochondria are the cytoplasmic organelles responsible for breathing and the production of the energy necessary for cell survival. Mitochondria undergo morphological changes of fusion and fission depending on the energy needs of the cell. The regulation of the balance between these two processes is crucial for cell survival; an alteration in any of them could lead to cellular pathological conditions. [1]

Fission and fusion determine mitochondrial morphology. These fusion and fission processes are regulated by the Opa1 and Drp1 proteins respectively. Their cell concentrations vary depending on aspects such as oxidative stress, cell cycle, mitochondrial metabolism, etc. [2]

In mitochondrial fission, post-translational modifications such as phosphorylation, sumoylation, ubiquitination, s-nitrosylation and o-glcnacylation are necessary requirements for the functionality of Drp1 [3]. Activation occurs through its interaction with protein receptors of the external mitochondrial membrane such as Fis1, Mff, MIEF1 and MIEF2, in addition to the participation of others such as actin and cardiolipin. $[4,5,6]$. Drp1 is activated by forming a ring-shaped structure that attaches to the outer mitochondrial membrane that constricts [twists] and cleaves the mitochondria [7].

The endoplasmic reticulum interacts with Drp1 during mitochondrial fission, intervening in $88 \%$ of mitochondrial fissions that occur in the cell. The remaining $12 \%$ are mitochondrial divisions regulated by independent contact sites of the RE-mitochondria junction [3]. The contact sites of the external mitochondrial membrane are sites of high concentration of cardiolipin where the Fis1, Mff, MIEF1 and MIEF2 proteins are found that regulate the activation of Drp1 during the mitochondrial fission process [3].

The mitochondrial DNA encodes the proteins necessary to synthesize the subcellular complexes responsible for forming the electron transport chain. Defects in the mitochondrial DNA condition cellular levels of Drp1. Certain alterations in DNA lead to overexpression of Drp1 in the cell, inducing mitophagy as a quality control mechanism [8].

It has been shown that Drp1 is important for neuronal maturation and brain development, and for the survival of cerebellar Purkinje cells among other neuronal species [1], since inhibition of Drp1 
activity alters migration and typical morphology of the neuron [9]. In addition, it is postulated as a compensation mechanism for a neuronal lesion [10].

Drp1 seems to be a common factor in neurodegenerative diseases such as Huntington's disease, Alzheimer's, Parkinson's, etc. in which the fusion/fission balance is altered. Consequently, there is a morphological alteration of the mitochondria that favor the fission. These excessively fissioned mitochondria cannot meet the cellular energy demand, which triggers apoptotic processes characteristic of cell degeneration and the appearance of neurodegenerative pathology [11].

It is the overexpression of Drp1 that leads to a downregulation of fusion factors causing mitochondrial fission [12]. Inhibition of excessive mitochondrial fission through the mechanism of action of Drp1 delays apoptosis. This fact prevents the release of cytochrome c and, consequently, the intrinsic pathway of apoptosis is inhibited [13]. That is why the inhibition of Drp1 is proposed as an effective neuroprotection strategy [14].

Therefore, finding a control mechanism of Drp1 by which the physiological fusion/fission balance is restored is critical for proper cellular functioning in neurodegenerative diseases.

\section{MATERIAL AND METHODS}

\section{PubMed search}

To obtain the articles that make up this bibliometric study we conducted a search in the US National Library of Medicine of the National Institutes of Health (http://www.ncbi.nlm.nih.gov/pubmed/).

The search was performed with the PMC Advanced Search Builder tool, the terms used were drp1 AND Alzheimer disease and drp1 AND Parkinson disease. In addition, to avoid possible confusion with the name of the protein, the OR command was also used followed by Dynamin related protein 1 and DRP1 (Table 1). Among all the articles, only those that talk about a relationship between Drp1 and AD and PD were selected, because many articles found by the search only focused on one of the two terms and did not provide information that was consistent with the initial objective of the study.

Table 1. Pubmed searches performed in this study

Search: (((Alzheimer) AND drp1) OR Dynamin related protein 1) OR DRP1 Sort by:

Best Match Filters: Publication date from 2008/01/01 to 2019/12/31

Search: (((Parkinson) AND drp1) OR Dynamin related protein 1) OR DRP1 Sort by:

Best Match Filters: Publication date from 2008/01/01 to 2019/12/31

The date range of the publications is from $01 / 01 / 2008$ to $12 / 31 / 2019$. In this way, we tried to get the most relevant and recent articles in addition to searching enough years to clearly observe the evolution of the publications. Excluding years before 2008 was done because the relationship between Drp1 and AD and PD is recent and 11 years was thought sufficient to highlight the role of Drp1 in the 
pathogenesis of these diseases. All the bibliography using for this bibliometric study is in the supplementary document for AD and PD.

\section{Statistical analysis}

The graphs and statistical analysis were performed with the Microsoft Excel program in xlsx format. The articles were divided into two large groups, those that manifested a relationship between Drp1 and Parkinson's and a second group that related Alzheimer's with Drp1. These two large groups of articles were classified by year, also dividing them into articles in favor of the hypothesis and articles against it.

Finally, and to quantitatively demonstrate the validity of the articles, the JCR of each article was observed on the Web of Science (https://jcr.clarivate.com/) and the citation index of each article (https://apps.webofknowledge.com/).

With all the data already collected, we proceeded to compose a series of graphs to show the evolution of the number of publications, the JCR of the publications, in Parkinson's (Table 2) and in Alzheimer's (Table 4) and the citation index of the same per year, both in Parkinson's (Table 3) and in Alzheimer's (Table 5).

Table 2. The parameters: years (from 2008 to 2019), articles in favor of the hypothesis: Drp1 is required for mitochondrial fission in PD; the medium JCR the articles in favor of the hypothesis, articles against the hypothesis and the JCR of articles against the hypothesis

\begin{tabular}{ccccc}
\hline YEAR & IN FAVOR & $\begin{array}{c}\text { JJCR } \\
\text { (In favor 2018) }\end{array}$ & AGAINST & $\begin{array}{c}\text { IJCR } \\
\text { (Against 2018) }\end{array}$ \\
\hline 2008 & 1 & 9,43 & 0 & 0,00 \\
2009 & 1 & 5,33 & 0 & 0,00 \\
2010 & 3 & 5,93 & 0 & 0,00 \\
2011 & 6 & 7,10 & 1 & 4,77 \\
2012 & 6 & 6,25 & 0 & 0,00 \\
2013 & 5 & 5,48 & 1 & 6,68 \\
2014 & 7 & 5,38 & 1 & 6,34 \\
2015 & 6 & 5,89 & 0 & 0,00 \\
2016 & 6 & 5,93 & 0 & 0,00 \\
2017 & 3 & 4,06 & 0 & 0,00 \\
2018 & 6 & 3,97 & 0 & 0,00 \\
2019 & 3 & 5,83 & 0 & 0,00 \\
\hline Total & 53 & & 3 & \\
\hline
\end{tabular}


Table 3. The parameters: years (from 2008 to 2019), the medium JCR of articles in favor of the hypothesis: Drp1 is required for mitochondrial fission in PD; the JCR of articles against the hypothesis, the number of citations of articles in favor of the hypothesis and the number of citations of the articles against the hypothesis

\begin{tabular}{ccccc}
\hline YEAR & $\begin{array}{c}\text { JJCR } \\
\text { (in favor 2018) }\end{array}$ & $\begin{array}{c}\text { ECITES } \\
\text { (in favor 2018) }\end{array}$ & $\begin{array}{c}\sum \text { JCR } \\
\text { (Against 2018) }\end{array}$ & $\begin{array}{c}\text { CCITES } \\
\text { (Against 2018) }\end{array}$ \\
\hline 2008 & 9,43 & 591 & 0,00 & 0 \\
2009 & 5,33 & 543 & 0,00 & 0 \\
2010 & 5,93 & 273 & 0,00 & 0 \\
2011 & 7,10 & 560 & 4,77 & 291 \\
2012 & 6,25 & 587 & 0,00 & 0 \\
2013 & 5,48 & 556 & 6,68 & 73 \\
2014 & 5,38 & 293 & 6,34 & 165 \\
2015 & 5,89 & 115 & 0,00 & 0 \\
2016 & 5,93 & 148 & 0,00 & 0 \\
2017 & 4,06 & 38 & 0,00 & 0 \\
2018 & 3,97 & 28 & 0,00 & 0 \\
2019 & 5,83 & 0 & 0,00 & 0 \\
\hline Total & & 3732 & & 529 \\
\hline
\end{tabular}

Table 4. Comparison of JCR and number of publications between in favour and against articles per year of Drp1 and AD

\begin{tabular}{cccccccc}
\hline YEAR & $\begin{array}{c}\text { TOTAL } \\
\text { ARTICLES }\end{array}$ & $\begin{array}{c}\text { JCR } \\
\text { (year } \\
\text { published) }\end{array}$ & JCR (2018) & IN FAVOUR & $\begin{array}{c}\text { JCR } \\
\text { (IN } \\
\text { FAVOUR) }\end{array}$ & AGAINST & $\begin{array}{c}\text { JCR } \\
\text { (AGAINST) }\end{array}$ \\
\hline 2008 & 2 & 7,54 & 6,67 & 2 & 7,54 & 0 & 0,00 \\
2009 & 3 & 14,43 & 17,86 & 3 & 14,43 & 0 & 0,00 \\
2010 & 3 & 3,97 & 3,66 & 2 & 3,82 & 1 & 4,26 \\
2011 & 2 & 8,24 & 6,32 & 2 & 8,24 & 0 & 0,00 \\
2012 & 5 & 7,62 & 6,60 & 5 & 7,62 & 0 & 0,00 \\
2013 & 8 & 7,25 & 6,77 & 8 & 7,25 & 0 & 0,00 \\
2014 & 4 & 4,74 & 4,84 & 4 & 4,74 & 0 & 0,00 \\
2015 & 7 & 4,74 & 5,53 & 7 & 4,74 & 0 & 0,00 \\
2016 & 9 & 5,33 & 5,24 & 9 & 5,33 & 0 & 0,00 \\
2017 & 7 & 5,94 & 6,09 & 7 & 5,94 & 0 & 0,00 \\
2018 & 10 & 4,33 & 4,33 & 10 & 4,33 & 0 & 0,00 \\
2019 & 6 & 5,61 & 5,61 & 6 & 5,61 & 0 & \\
\hline
\end{tabular}


Table 5. Comparison of citation index between publications in favour and against per year

\begin{tabular}{cccc}
\hline YEAR & TOTAL CITES & CITES IN FAVOUR & CITES AGAINST \\
\hline 2008 & 653 & 653 & 0 \\
2009 & 1192 & 1192 & 0 \\
2010 & 204 & 117 & 87 \\
2011 & 380 & 380 & 0 \\
2012 & 441 & 441 & 0 \\
2013 & 1013 & 1013 & 0 \\
2014 & 285 & 285 & 0 \\
2015 & 156 & 156 & 0 \\
2016 & 250 & 250 & 0 \\
2017 & 98 & 98 & 0 \\
2018 & 46 & 46 & 0 \\
\hline Total & & 4631 & 87 \\
\hline
\end{tabular}

\section{RESULTS}

\section{The number of articles remains constant over time}

After the search conducted in the US National Library of Medicine of the National Institutes of Health (http://www.ncbi.nlm.nih.gov/pubmed/), we classified the number of publications per year, in order to see the number of articles in each year, and see the trend of publication in order to observe the interest in the relationship of Drp1 with AD and PD.

\section{Drp1 in Parkinson's Disease Publications}

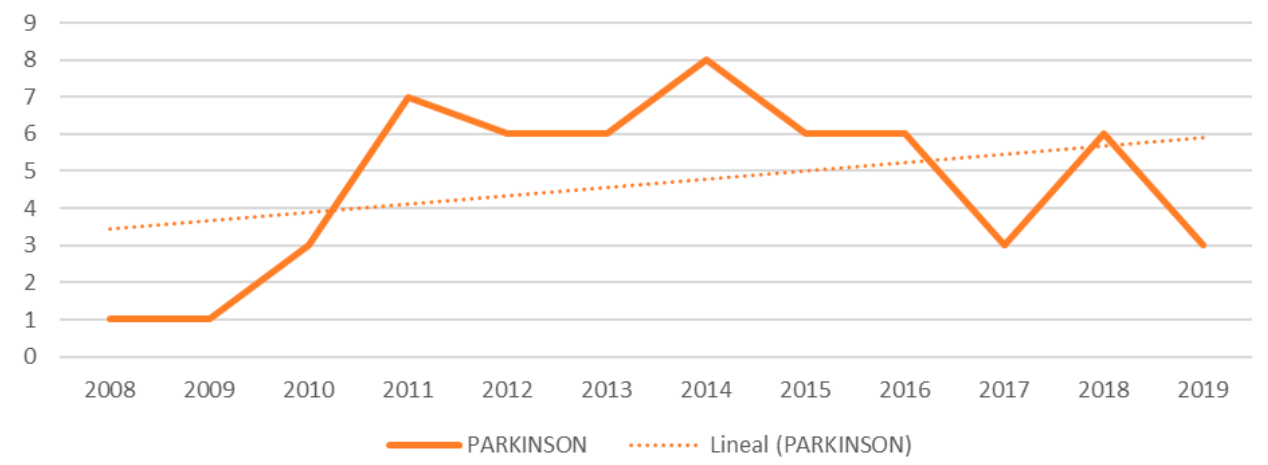

Fig. 1. In the axis of the ordinates the number of revised articles, in the axis of abscissas years from 2008 to 2019. Table 1 represents the progression of research of Drp1 in Parkinson's disease 
In the case of Parkinson, the graph (figure 1) shows a positive trend line, so that the increase in the number of publications over time indicates that there has been a growing interest in this field of research. Similarly, in the case of Alzheimer's (figure 2) it can be seen that the number of articles increases over time and is mainly reflected in the trend line that is clearly rising.

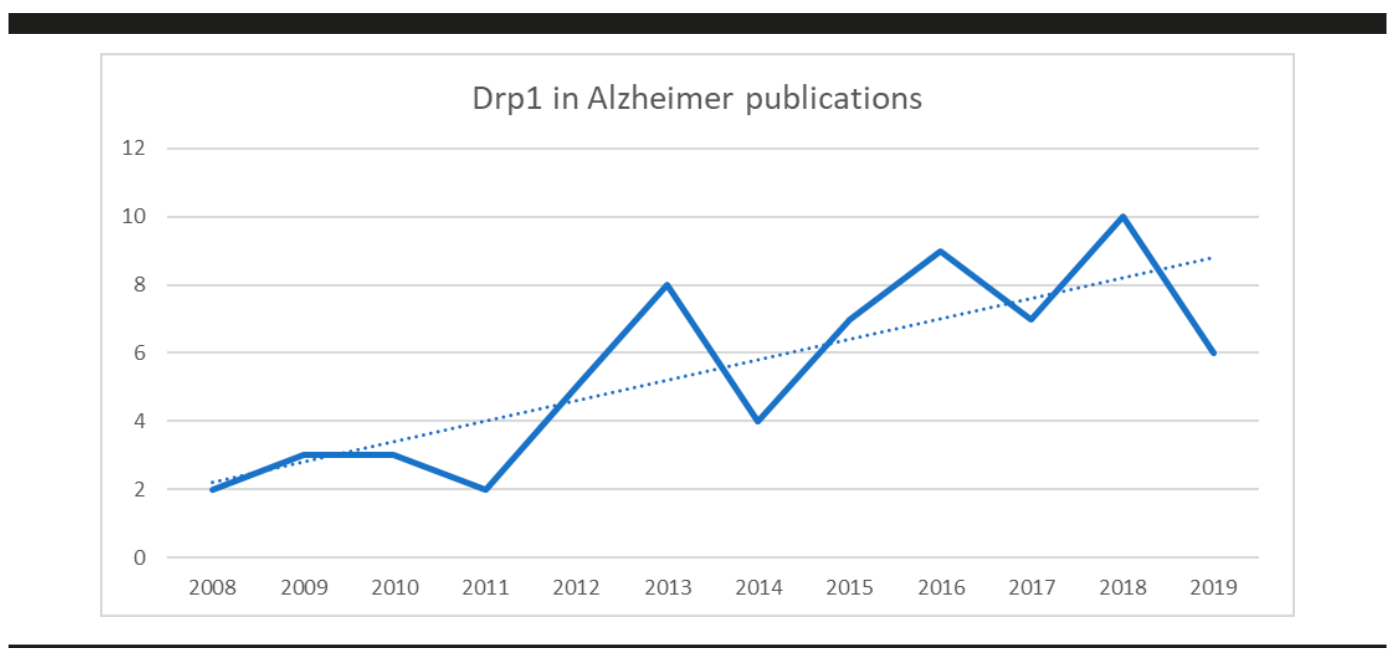

Fig. 2. Total number of publications of the relationship between Drp1 and AD per year between 2008 and 2019, based on PubMed database

\section{The average JCR of the journals remained stable over time}

To verify that the interest had not diminished over time we made a comparison of the average JCR between the year of publication and the average JCR at present of the magazine, considering that in some cases the JCR of 2019 was not available and we used the value for 2018 as an estimate.

For PD, Figure 3 shows that the value of the JCR has not changed excessively, indicating that the interest and relevance of this protein in the pathology have remained constant.

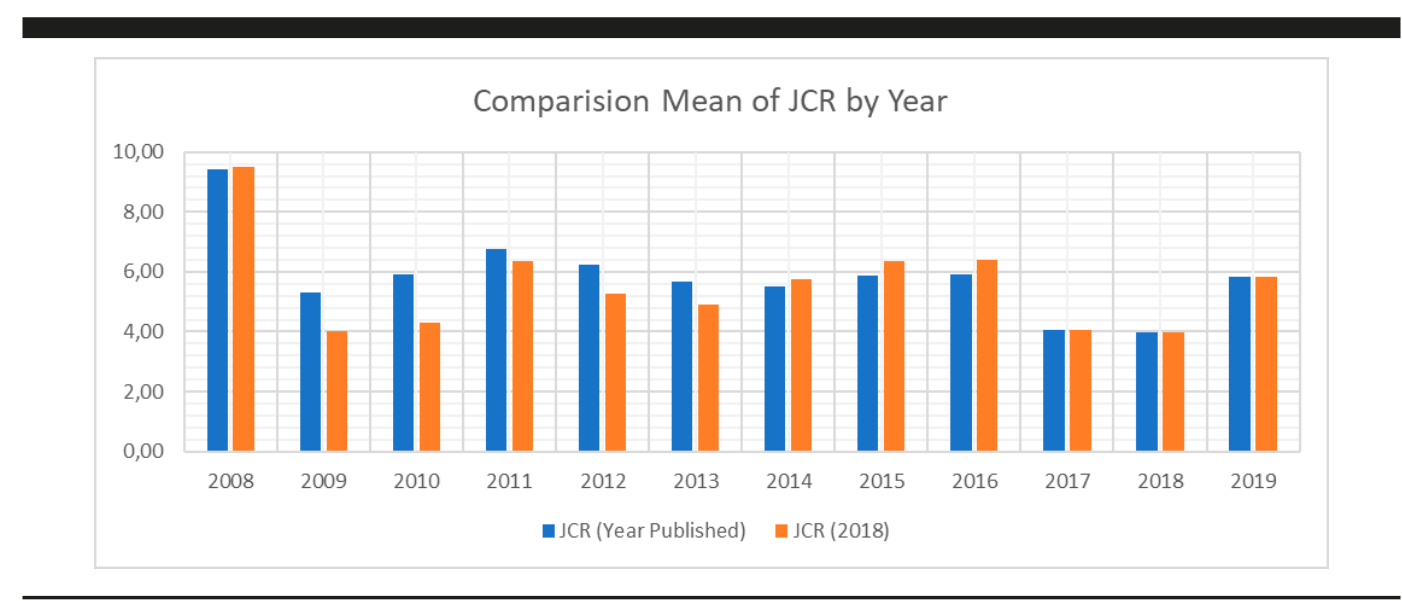

Fig. 3. In the axis of ordinates, the medium JCR revised articles, in the axis of abscissas years from 2008 to 2019. Table 2 shows the evolution of the absolute JCR 
It should be noted that the average JCR value of the year of publication was slightly higher than the current year except for 2008, 2014, 2015 and 2016 where it was slightly lower.

note that in 2017 and 2018 both JCR values were equal because they are data obtained from the same year, and that the 2019 values are an approximation, taking the JCR of these articles in 2018 as a reference.

In the case of $\mathrm{AD}$ in Figure 4, in the last 5 years of study, the value of the average JCR did not vary significantly between the years in which the publication was made and the value of the average JCR until 2019. The average JCR of each year was 6.64 in the year of publication, and 6.63 for 2018, which implies minimal variations in interest in the relationship of Drp1 with AD.

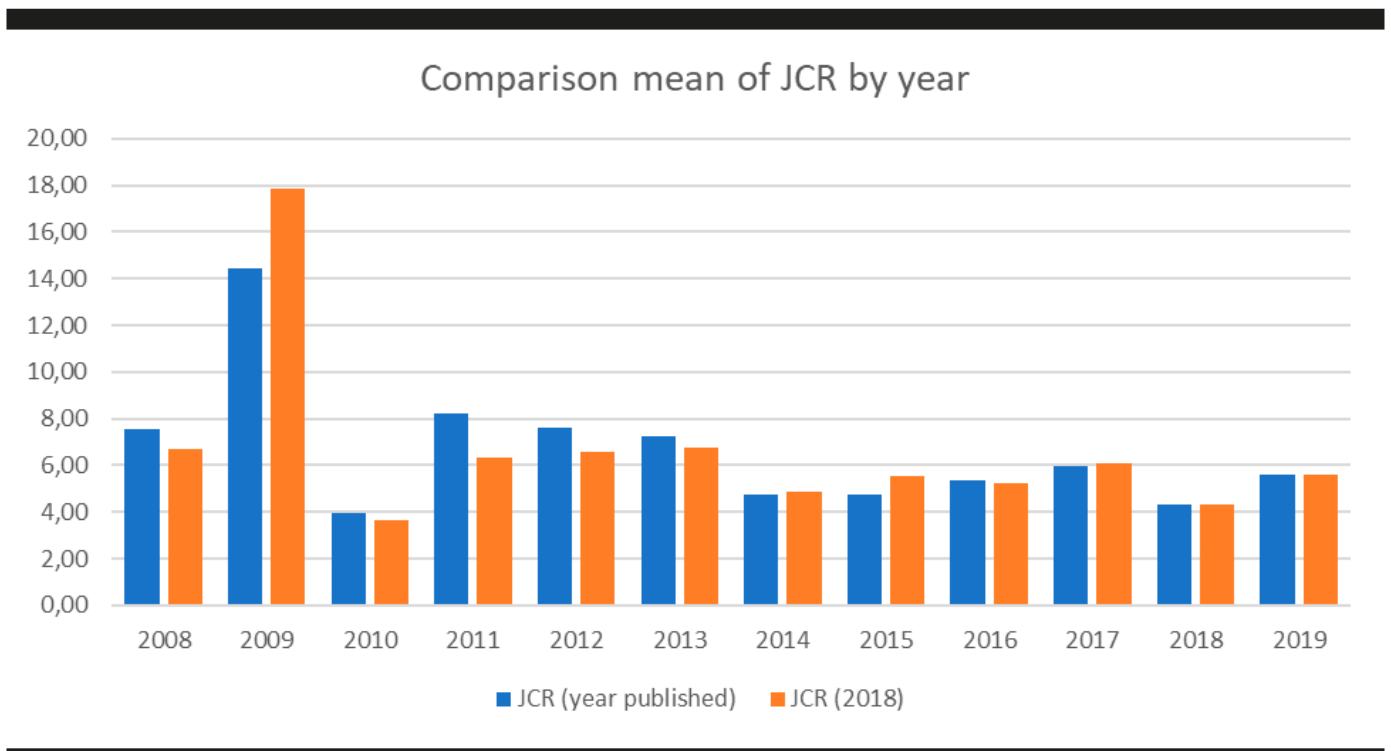

Fig. 4. Evolution of JCR publications of Drp1 and AD per year, between 2008 and 2019, JCR based on Web of Science database

\section{The average JCR and the number of articles are stable over the years}

The evolution over time of the average JCR and the number of publications are shown by plotting them on the same graph.

For the case of PD (figure 5), the number of published articles has not changed significantly over time. Note a higher number of articles published in 2014 but the average value of JCR did not change.

The value of the JCR has remained stable. A relative minimum was observed in 2017, down to 4.06 , possibly because only 3 articles were published that year. We also observed a notable relative maximum in 2011 where 6 articles with an average JCR value of 7.1 were published. 


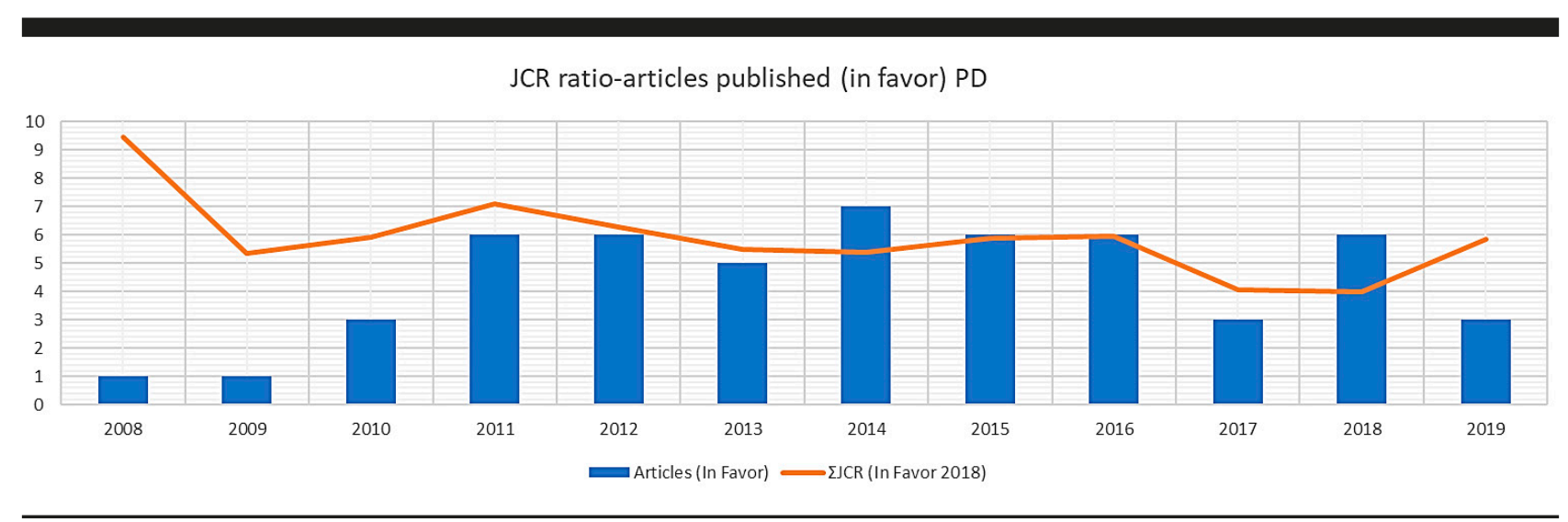

Fig. 5. In the ordinated axis the JCR medium, in the axis of abscissas years from 2008 to 2019. Figure 5 shows the relationship between JCR year of publication with articles published in the same year.

The graph also shows a maximum in 2008 that deviates from the average JCR with a value of 9.4, corresponding to the only article published that year. This data is an absolute value and not an average as in the other years. This article was not been removed from the study because it met the inclusion/ exclusion criteria.

In 2009 there is also a single article but the JCR value was normal. This was also not ruled out because it met the inclusion/exclusion criteria.

For AD, as shown in Figure 6 and Figure 1, the number of items per year show the evolution of the average JCR and its relationship to the number of articles per year. It can be seen, as in previous graphs, that as of 2013 the number of publications stabilized with an average of 7 articles per year. In those years the average JCR showed small fluctuations in value, very different from the first years of study in which the relationship between Drp1 with AD was not so clear.

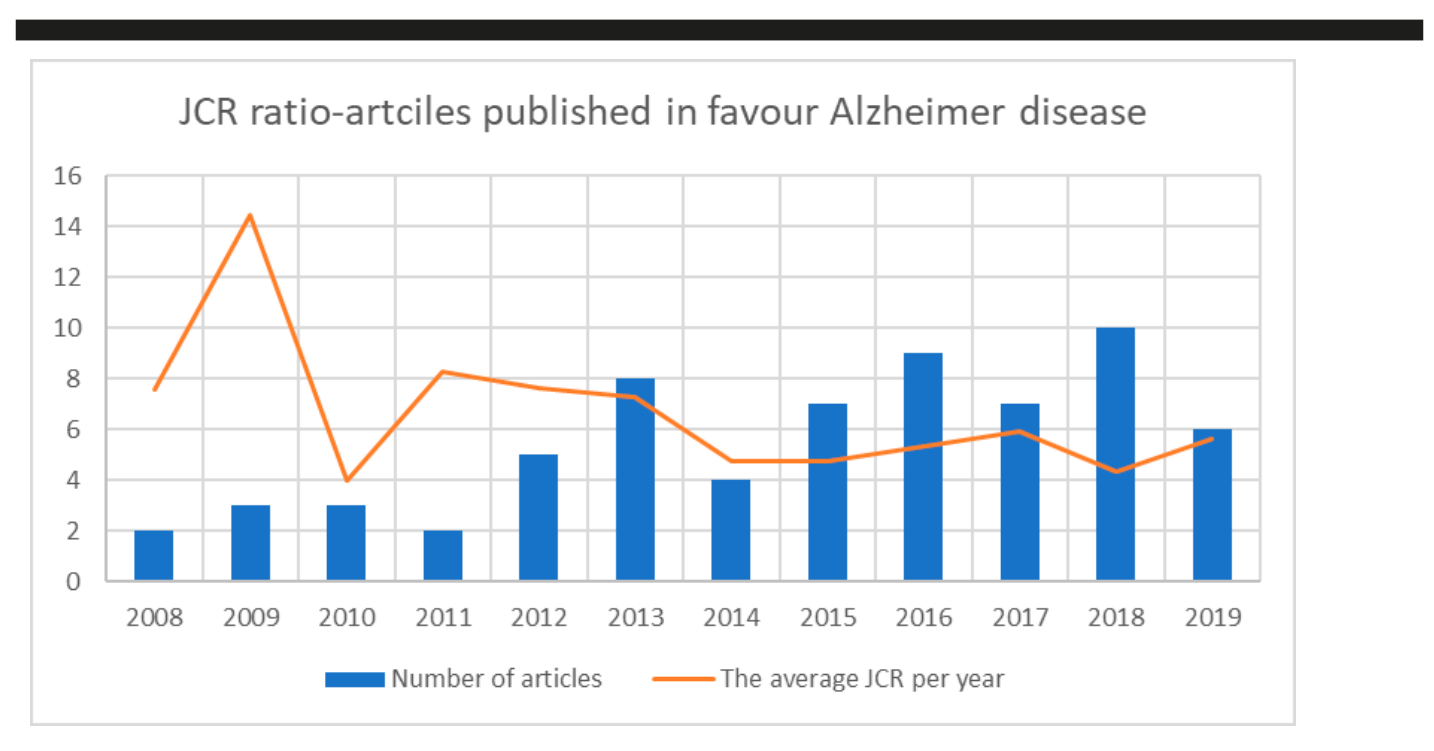

Fig. 6. Comparison between the evolution of JCR per year between 2008 and 2019 and the number of publications per year between 2008 and 2019, based on Web of Sciences and PubMed databases 


\section{The initial hypothesis of the study was supported by most articles}

The figures do not include one that compares the number of articles in favor and the articles against the thesis that there is a relationship of Drp1 with AD and PD. This is because the numbers of articles against the hypothesis were very small; 1 in the case of $\mathrm{AD}$ and 3 in the case of PD and the citation index was very low compared to articles in favor for both pathologies. These data can be seen in Tables 2, 3, 4 and 5.

\section{DISCUSSION}

The molecular basis of AD and PD is completely unknown, however, both in AD and PD, mitochondrial fission plays a fundamental role in the pathogenesis of both diseases.

At present the detailed molecular mechanisms in both pathologies are unknown. However, we suggest that both pathologies end up converging on an affectation of mitochondrial morphology, reflected in an increase in fission. This is why Drp1 is proposed as a possible therapeutic target because it is the main protagonist of mitochondrial fission.

In both pathologies, mitochondrial morphology varies due to the fusion/fission imbalance caused by overexpression of Drp1.

The role of Drp1 in neurodegenerative diseases is known. For its activation, protein binding in the external mitochondrial membrane is necessary, as mentioned above. The direct interaction of proteins properly related in AD and PD with Drp1 has not been described, so Drp1 is postulated as a key piece between the initial pathogenesis of these diseases and the intrinsic apoptosis entailed by the characteristic neurodegeneration of these diseases. We suggest Drp1 as a central molecule in the pathogenesis that could delay the natural history of PD and AD.

It is known that $12 \%$ of mitochondrial fissions are performed independently of Drp1. This fact can explain the small but significant number of articles against the hypothesis proposed here. Even so, we consider that this fact does not detract from the role of Drp1 in fission and its potential capacity to slow the progression of the disease.

Figure 2 shows the number of articles published per year. An increase in the number of published articles on the relationship between Drp1 and Alzheimer's disease over the years can be observed, showing that the interest in the relationship of Drp1 with Alzheimer's disease is growing.

Figure 4 compares the average JCR of the journals in the year of publication of the article and the value of the average JCR at present. Variations can be seen in the early years of the study that are probably due to the lack of information on the relationship of Drp1 with Alzheimer's. However, in the last 6 years of our study, between 2013 and 2019, the values of the average JCRs do not vary significantly and remains stable over time. From this, we conclude that the interest of Drp1 in relation to the average value of the JCR remains.

Table 4 shows the different data of the study, both the number of articles and the value of the average JCRs per year, which were used to carry out our study.

Table 5 compares the citation index of the articles for and against. The citation index of the articles in favor is much higher than that of the articles against, so we can conclude that the articles in favor have more weight than the articles against in the relationship between Drp1 and Alzheimer. 
Figure 6 shows the relationship between the average JCR value of the articles and the number of publications throughout the years of the study. Although it is clearly seen that in the years 2008 and 2009 there is a higher value of the average JCR, because there was a greater interest and less information about the relationship between Drp1 and Alzheimer, the number of publications in those years were two and three respectively, less than the number of articles published as of 2012.

As of that year, the average number of publications was between six and seven articles per year with an average JCR of 6,63. Therefore, from 2012 to 2019, both the number of publications and their average JCR value remained stable.

We can conclude that the interest in the relationship between Drp1 and Alzheimer's remained stable in terms of the value of the average JCR and the increase in the number of publications.

Several theories are postulated on the relationship between the pathogenesis of Alzheimer's and Drp1. The most accepted theory is based on the fact that the accumulation in the neurons of the amyloid B plates causes an increase in oxidative stress in the neuron. This oxidative stress produces a fission/fusion imbalance at the mitochondrial level in favor of fission, which is due to an overexpression of Drp1 and as a consequence will produce the fragmentation of the mitochondria, and finally the early apoptosis of the neurons that is characteristic of the diseases neurodegenerative as is Alzheimer's. Another theory would involve the interaction between Tau and Drp1, although the relationship between the two is not clear and more experimental evidence would be required.

That is why Drp1 is postulated as a possible therapeutic target, and that its inhibition or regulation through other molecules or modifications could lead to a new strategy to treat Alzheimer's. However, it is necessary to advance the molecular mechanisms of control of Drp1 for the compression of the evolution of AD.

Drp1 acts on Parkinson's disease producing a dysregulation of mitochondrial fission/fusion homeostasis resulting in oxidative stress that eventually degenerates these neurons. To do this, Drp1 interacts with several proteins related to this pathology such as PINK1, Parkin, $\alpha$ - synuclein and DJ-1.

Regarding the results provided by the tables, we can observe in Table 1 that there has been a growing interest in the pathology and its relationship with Drp1. This may be due to several reasons: Greater financing, better means for obtaining results, etc.

Figure 3 shows that the average impact of this protein over time has been maintained, being in the JCR value at an average of 6 . With this and the values obtained in Figure 1, we suspect that it is a possible influence of Drp1 on Parkinson's disease.

Table 2 and Table 3 are the data that were used in this bibliometric review.

Regarding Table 3, we see that the articles in favor of the hypothesis were cited more than those against it, from which it is inferred that there is a correlation between Drp1 and neurodegenerative pathogenesis such as PD or AD. Although the number of citations of the articles that are against the hypothesis is significant depending on its impact index.

This fact does not detract from the role of Drp1 in the neurodegenerative pathogenesis, this is observed in Figure 5, which shows us the articles that favor the hypothesis (Drp1 is necessary for mitochondrial fission in the pathology of PD) and his JCR. These data suggest that this theory has been accepted in the scientific community, being able to present DRP1 as a possible therapeutic target for Parkinson's disease. 
Although there is some consensus on the role of Drp1 in the pathogenesis of PD, there is some controversy about the individual importance of each of the typical proteins mentioned above (PINK1, Parkin, $\alpha$ - synuclein and DJ-1) that are altered in the illness.

There are several hypotheses in this regard: One of them is that it is these proteins that provide quality control of Drp1 and Opa1; another is that there are several fission pathways, one mediated by Drp1 / Opa1, another mediated by PINK1 / Parkin in which they would function as the functional homologues of Drp1 and Opa1, and another in which these four proteins would participate.

It is a common point that, despite the presence of other factors, is Drp1 the central molecule that causes the deregulation of mitochondrial morphology in the pathogenesis of PD.

Therefore, we propose Drp1 as a possible therapeutic route that delays the effects of apoptosis and thus delay the progression of the disease. To elucidate these results, it would be convenient to carry out future research that clarifies the interactions of Drp1 and other proteins with the pathogenesis of Parkinson's disease.

\section{CONCLUSIONS}

After analyzing the exposed data, Drp1 has great potential as a possible therapeutic target in AD and PD diseases since the data shown in the study provide favorable arguments for the hypotheses raised.

Drp1 plays a fundamental role in the pathogenesis of Alzheimer's and Parkinson's disease.

After highlighting the role of Drp1 in the regulation of homeostasis of mitochondrial morphology in patients with $\mathrm{PD}$ and $\mathrm{AD}$ disease, we can conclude that Drp1 represents a new therapeutic strategy for addressing diseases such as PD and AD

\section{ACKNOWLEDGMENT}

The authors thank support from Universidad Católica de Valencia San Vicente Mártir

\section{LITERATURE CITED}

[1] Oettinghaus B, Schulz JM, Restelli LM, Licci M, Savoia C, Schmidt A, ... Frank S. Synaptic dysfunction, memory deficits and hippocampal atrophy due to ablation of mitochondrial fission in adult forebrain neurons. Cell Death and Differentiation. 2016;23(1):18-28. [Pubmed].

[2] Wang X, Su B, Lee H, Li X, Perry G, Smith MA, et al. Impaired Balance of Mitochondria Fission and Fusion in Alzheimer Disease. J Neurosci. 2009;45(6):788-802. [Pubmed].

[3] Pagliuso A, Cossart P, Stavru F. The ever-growing complexity of the mitochondrial fission machinery. Cellular and Molecular Life Sciences. 2018;75(3):355-374. [Pubmed].

[4] Atkins K, Dasgupta A, Chen KH, Mewburn J, Archer SL. The role of Drp1 adaptor proteins MiD49 and MiD51 in mitochondrial fission: implications for human disease. Clinical Science. 2016;130(21):1861-1874. [Pubmed]. 
[5] Rehklau K, Hoffmann L, Gurniak CB, Ott M, Witke W, Scorrano L, ... Rust MB Cofilin1-dependent actin dynamics control DRP1-mediated mitochondrial fission. Cell Death and Disease. 2017;8(10):e3063. [Pubmed].

[6] Kameoka S, Adachi Y, Okamoto K, Iijima M, Sesaki H. Phosphatidic Acid and Cardiolipin Coordinate Mitochondrial Dynamics. Trends Cell Biol [Internet]. 2017;xx:1-10. [Pubmed].

[7] Basu K, Lajoie D, Aumentado-Armstrong T, Chen J, Koning RI, Bossy B, ... Rouiller I Molecular mechanism of DRP1 assembly studied in vitro by cryo-electron microscopy. PLoS ONE. 2017;12(6):1-21. [Pubmed].

[8] Kiriyama Y, Nochi H. Intra- and Intercellular Quality Control Mechanisms of Mitochondria. Cells. 2017;7(1):1. [Pubmed].

[9] Kim HJ, Shaker MR, Cho B, Cho HM, Kim H, Kim JY, et al. Dynamin-related protein 1 controls the migration and neuronal differentiation of subventricular zone-derived neural progenitor cells. Sci Rep [Internet]. 2015;1-13. [Pubmed].

[10] Kiryu-Seo S, Tamada H, Kato Y, Yasuda K, Ishihara N, Nomura M, ... Kiyama H. Mitochondrial fission is an acute and adaptive response in injured motor neurons. Scientific Reports. 2016;6:1-14. [Pubmed].

[11] Chaturvedi RK, Beal MF. Mitochondrial diseases of the brain. Free Radic Biol Med [Internet]. 2013; 63:1-29. [Pubmed].

[12] Alaimo A, Gorojod RM, Beauquis J, Muñoz MJ, Saravia F, Kotler ML. Deregulation of mitochondria-shaping proteins Opa-1 and Drp-1 in manganese-induced apoptosis. PLoS One. 2014;9(3). [Pubmed].

[13] Estaquier J, Arnoult D. Inhibiting Drp1-mediated mitochondrial fission selectively prevents the release of cytochrome c during apoptosis. Cell Death Differ. 2007;14(6):1086-94. [Pubmed].

[14] Grohm J, Kim SW, Mamrak U, Tobaben S, Cassidy-Stone A, Nunnari J, et al. Inhibition of Drp1 provides neuroprotection in vitro and in vivo. Cell Death Differ [Internet]. 2012;19:1446-58. [Pubmed]. 